\title{
Concept Solution Generation for Product Innovation under User Fuzzy Semantic Requirements
}

\author{
WANG Zuojie $^{1}$, LIU Libing ${ }^{2}$, TAN Zhihong ${ }^{3}$, YANG Zeqing ${ }^{2 *}$, LIU Yanan $^{2}$, ZHANG Song $^{1}$ \\ ${ }^{1}$ Environmental Protection subcompany, Sinoma Technology \& Equipment Group CO.,LTD., \\ Tianjin 300400, China \\ ${ }^{2}$ School of Mechanical Engineering, Hebei University of Technology, Tianjin, 300130, China \\ ${ }^{3}$ School of Environmental and Chemical Engineering, Nanchang University, Nanchang 330031, China \\ * Corresponding author's Email: yzq82@163.com
}

\begin{abstract}
According to the classification of user requirements in Kano model, theory of technology evolution was adopted to determine the future evolution route of product and predict possible structure state for the future technology, in order to mine the user's potential requirements. In view of the fuzzy and uncertainty of users' potential requirements, the fuzzy semantic model was adopted to describe the qualitative user requirements in order to standardly form the user requirements concept sets with functional attributes in different requirement categories, so as to guide the designers to create the innovative solutions along the right direction and then achieve the product rapid innovatio$\mathrm{n}$ design. Taking the Pulse-Jet bag filter as an example, the case analysis shows that this method can effectively be applied to obtain new product innovation concept solution, and provide theoretical support for the mapping between subsequent innovation concept solutions and innovation function solutions and innovation structure solutions, and also provide the model architecture for innovation droved new product digital prototype design.
\end{abstract}

Keywords: Fuzzy semantic model; Theory of technology evolution; Product innovation; Concept solution; Bag filter

\section{Introduction}

Elicitation and predicting user requirements are the front-end process of product conceptual design and the direct source of the conceptual design target, which have important effects on the development of new products [1]. Reference[2] and [3] proposed that user requirements were confirmed through market pull and technology driven mode in product innovation design process. Reference [4-8] analyzed user requirements in product research, design and manufacturing phases through QFD, and provided the model architecture for determining the final importance ratings of user requirements. Reference [9] established the common product requirements information model adopting the formal description language EXPRESS-G through analyzing the requirements of product structure and development process. Reference [10] proposed the decision support system which considered the market demand and its influence factors, customer satisfaction and product function properties to predict user requirements. The elicitation and prediction of user requirements from different angles by different methods and tools were researched, however, it is not well solved that how to effectively extract, transform, and describe the users' potential and dynamic requirements information.

In order to adapt the market needs and enhance the competitiveness of the enterprise, according to the classification of user requirements in Kano model, technology evolution theory was adopted to determine the future evolution route of product, predict possible structure state for the future technology and excavate the users' potential and dynamic requirements. In view of the fuzziness and uncertainty of users' potential requirements, the fuzzy semantic model was adopted to describe the qualitative user requirements to establish the concept sets with 
functional attributes in different requirement types. Designers can understand and predict the users' potential requirements easily, and they can follow the method to create the innovative solutions in right direction and then achieve the product rapid innovation design.

\section{Elicitate and Predict of User Requirements}

\subsection{Elicitation method of user requirements}

In the fierce market competition, the enterprise should be timely to understand the user requirements of products and develop the products meeting the demand of market. However, the user requirements are the dynamic processing influenced by consumer groups, environment, time, region and even political factors, which will change with time, technology, market. User requirements were divided into three types: basic type, expected type and interested type in Kano model [11]. The basic requirement's type is considered by the user that it should be the basic functions in the product; the expected requirement's type generally is the demand for the quality of the product, which realizes the better in the products, users will be much more satisfied with the quality of the product; the interested requirement's type refers to the potential demand that user unexpected, the degree of user satisfaction will greatly enhance, according to the interested requirement to make small improvements and innovate additional features on product features and functions. So it is the critical step of the product concept innovation design to find and extract the potential user requirements.

The elicitation methods of user requirements include user surveys, network consultation, market research and statistic analysis, etc..[11] The methods can analyze the change of user requirements through the product functional requirements in the market survey, and can judge the trends of product functional requirements to timely adjust product strategy and improve the quality of products and services. But they get arbitrary products requirements information. In addition, the development of new products in the enterprise should predict the product user requirements.

\subsection{Predict potential user requirements adopting technology evolution routes}

Altshuller found the technology evolution theory through the analysis of world patent library, and proposed technology evolution patterns [12]. Each evolution pattern has several evolutionary routes, and every evolution route describes the state sequence of the product core technology from the characteristics of structural evolution, namely the course of product evolution along the evolution route is the transfer process between product core technology, it may be the improvement of the performance limitation, or cost reduction, or functio$\mathrm{n}$ increasing.

The knowledge provided by the requirements evolution can find the new evolution trend of the product, and then the new function, new structure and new technology of the product were excavated to predict the potential requirements of the future products. There are ten kinds of modes in product evolution: simplification, micro-adjustment, flexibility, surface parametrization, multiple-dimension, harmonization, integration, automation, intellectualization, idealization. Simplification referred to the evolution of the system from simple to complex, and then from complex to simple; micro-adjustment referred to the evolution of the system from the macroscopical object to the microscopic objects; flexibility referred to the system evolution towards the direction of increasing the dynamic; surface parametrization referred to the evolution of the system geometrical shape; multiple-dimension referred to the system evolution towards the direction of increasing the dimension; harmonization referred to the system evolution towards the direction of the harmony of different parts; integration referred to the system evolution towards the direction of the supersystem; automation referred to the system evolution towards the direction of increasing the controllability; intellectualization referred to the system evolution towards the direction of reducing human intervention; idealistic referred to the system evolution towards the direction of increasing the idealized level [13]. Each mode has multiple evolution routes, The application of evolution modes and evolution routes can predict potential users requirements.

\section{Description Method of User Requirements}

\subsection{Fuzzy semantic model}

In natural language, language can be seen as the symbol system that the symbol is taken as word, which is composed by syntax and semantics words to express all kinds of things, ideas, behavior, state, and the feelings in the subjective and objective world. The algorithmic language of computer is the typical symbol system, which uses the certain symbols to represent machine movements and the state of the treated object, and it is the tool that people command on the machine. Most of the words 
expressing the user requirements for products are fuzzy words and phrases, in order to describe the fuzzy phenomenon in natural language quantitativel$y$ and formally, the language variables are introduced.

Language variables can be represented as ( $x$, $T(x), U, G, M)$, where $x$ is the variable; $T(x)$ is the word set, namely the set of the word that describe variables; $U$ is the domain of discourse of the basic variables, connecting with the basic variable $u ; G$ is the grammar rule, which generates the word set; $M$ is the semantic rule, which is the mapping function from the domain of discourse $U$ to $T(x)$ [14]. Thus, a language variable always link to the two rules, one rule is the grammar rules $G$, which illustrates to generate the every word of language value set $T(x)$ through analyzing the grammatical structure of language value; the other rule is the semantic rules $M$, which refers to the algorithmic rule about quantitative calculation of language value semantics. The fuzzy semanteme of the language value $T(x)$ for the language variable $x$ is a fuzzy set of the domain of discourse $U$, generally denoted by $M(T)$, the membership degree of elements in $\mathrm{U}$ is given by the membership function represented the fuzzy set, the selection of membership function is the critical step, which is determined gerenally by experience or statistical mathematical methods.

\subsection{Establish the fuzzy semantic model of user qualitative requirements}

According to the classification of user requirements in Kano model, various product demand groups with different functional properties are classified by user requirements according to different demand types and weights [15], where $B$ is basic requirement,
$E$ is expected requirement, $I$ is interested requireme$\mathrm{nt}$, each kind of requirement contains the clear quantitative requirement and fuzzy qualitative requirement description, the semantic information of clear quantitative requirement can be described with classic set, the set is the whole of the things with the particular attribute, in which contains a number of elements, the elements in the same set have common properties. In the classical set, "1" is represented the element belongs to a set, otherwise, it represents with " 0 ", so the two values " 1 "and "0" are generally taken in the classic set.

A series of text message language variable is generally used to describe the product requirements and design customized information towards the user qualitative demand, this not only depends on the degree of users' psychological cognition to the product, but also depends on the degree of designer's understanding to semantic information. Semantic information plays a decisive role in the relationship of the product-user-designer, however, the expressions of the semantic is often fuzzy and subjective description, the product symbol itself is the system composed of a variety of elements in a particular structure, which is a special symbol of information conversion, transmission and feedback between the product technology function and product structure taken as a medium of communicating message between designer and user. Fuzzy semantic concept and basic model were adopted to establish fuzzy semantic model for the user qualitative requirements, as shown in Fig.1. The product symbols were taken as the carrier of information, the object information firstly was encoded by the designer, and then was decoded by the user to realize the information transformation between designers and users. In the process of transformation between the designer, the

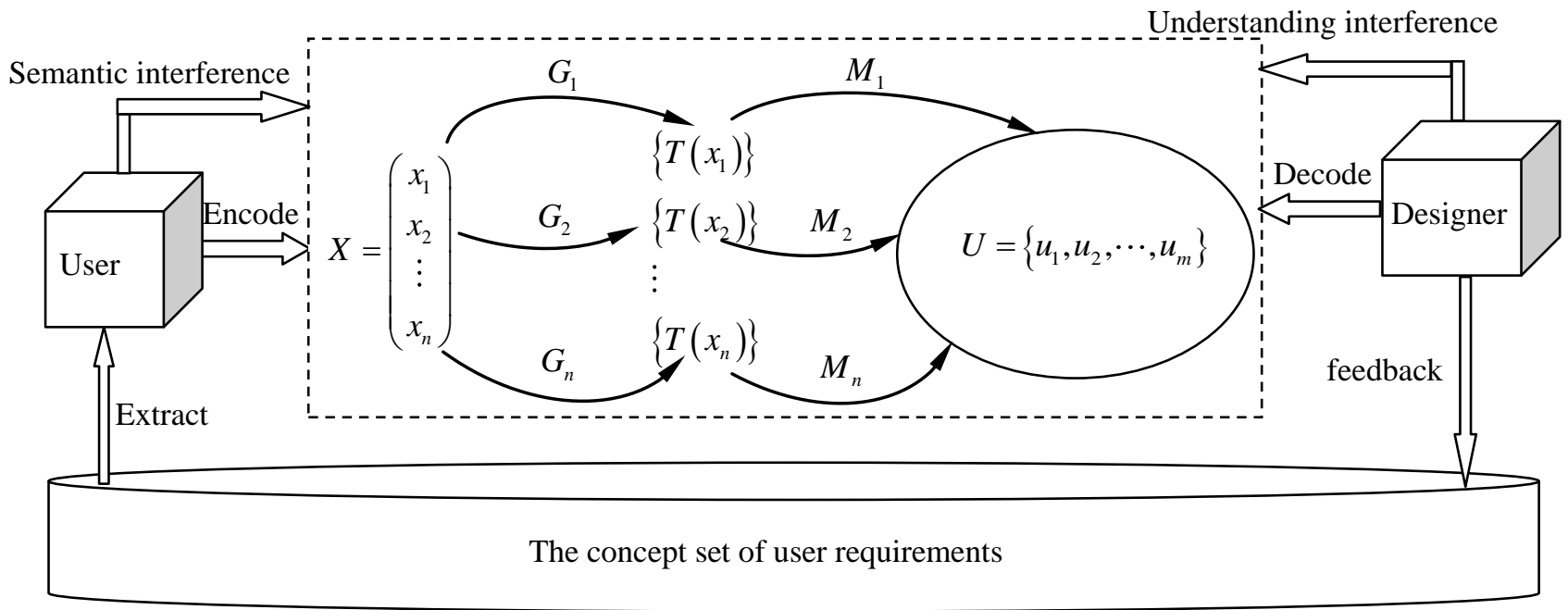

Figure 1 Fuzzy semantic model of user qualitative requirements. 
semantic model and users, product symbols will produce two deviations: designer encoding deviation and user decoding deviation, which will directly affect the efficiency of semantic transformation, but the model adjust the semantic interference and understand the interference parameters.

Thus, the semantic model of product symbols not only has the elements of the product characteristics reflected by the user dominant requirements, but also can reflect user implicit requirements information and the expression of the implied meaning of product information, in the process of semantic model, the user must predefine the attribute relationship hidden in the relationship object, and determine the variables of user qualitative demand, then establish the fuzzy set of user qualitative requirements, map and link to the relationship object, so as the associated semantics is built into the data model and the descriptive program of user requirements. The model was adopted to interpret the user requirements information in the product design through some symbol modeling, diagrams abstraction and some structure arrangement of semantic elements which can express the product meanings.

\subsection{Generate the concept set of product user requirements}

The normalized concept sets of the user requirements was expressed as Equation.1 based on the product symbols semantic model. The concept set includes "Terminology", "Category", "Factor", "Specification" and so on, which are defined in national standards. Each knowledge unit in the concept set of user requirements knowledge contains many types of relationships, such as "part_of" express the relationship between the part and the overall, "kind_of" express variety relations, "sibling_of" express two elements are the same category with transitivity and inheritance, "attribute_of" express attribute relationship.

$$
\text { Concept set }=\left\{\begin{array}{c}
\text { "Terminology" } \\
\text { "Category" } \\
\text { "Factor" } \\
\ldots \\
\text { "Specificat ion" }
\end{array}\right\}
$$

\section{Analysis of Application Example}

To verify the rationality and validity of the user qualitative requirements fuzzy semantic model in generating the user requirements concept set, taking the new products development of the bag filter as an example. The development process was summarized as follows. Firstly, the users' potential requirements for bag filter products were predicted adopting the technology evolution theory, the possible structure states of the products development were determined according to the trends of technology evolution; then, the fuzzy semantic model was established in view of the users potential requirements, and the concept set of user requirements for bag filter products was generated to guide designers to create new ideas and the concept solution of products innovation, which will provide the standardized model architecture for the mapping from the solution of subsequent innovation concept to innovation function and innovation structure.

\subsection{Predict the users' potential requirements for bag filter adopting technology evolution theory}

Bag filter was taken as the environmental protection equipment to collect dust by fibrous filter bag [17], it has the advantages of high efficiency of dust collection, strong adaptability for gas flow and dust concentration, stable operation,large processing flow, and reliable performance, etc. It accounts for about $70 \%$ of the total kinds of bag filters. 135 relevant patents of the main components and bag filters were searched in China's national Patent Office, we found that the pulse-jet bag filter structure development experienced several stages as follows.

Stage 1: Central jet pulsed bag filter;

Stage 2: Air box pulse bag filter;

Stage 3: Annular injection pulse bag filter;

Stage 4: Low pressure injection pulse bag filter;

Stage 5: Long-bag low-pressure pulse bag filter.

Through the patent analysis of the pulse-jet bag filter, the technology evolution mode of the pulse-jet bag filter towards the following three direction, and thus, which can predict the users' potential requirements for the bag filter in the future mainly focus on the following three aspects:

(1) Reducing the resistance of the equipment, injection pressure, and improving the filtering speed, deashing ability and dust-cleaning efficiency;

(2) Easy to demolite and install on-site;

(3) Increasing the length and life filter bag, and reducing the complexity of the filter bag structure.

\subsection{Establish the fuzzy semantic model of user qualitative requirements for bag filters}

The language variables of user qualitative requirements were determined for bag filters according to the above-mentioned technology evolution theory. A total of 11 language variables divided into "security", "filtration velocity", "deashing ability", "dust-cleaning efficiency", "equipment resistance", "length of 
the filter bag", "the service life of filter bag", "dust emission concentration" and "air volume", "consumption of steel in the structure", "stability and bearin$g$ of the structure". The evaluation set is the category set $V=\{$ classical category, improved category, innovation category\}. Firstly, the the membership degree $r_{i j}$ was considered that $u_{i}(i=1,2, \ldots, 11)$ as a single factor in the domain $U$ act on the category $v_{j}(j=1,2,3)$, so the single factor evaluation set of the $i$ th factor $u_{i}$ was $r_{i}=\left(r_{i}, r_{i 2}, r_{i 3}\right)$, and the total evaluation matrix $\mathrm{R}$ was composed by 11 variables' evaluation, that was $\mathrm{R}=\left(R_{i j}\right)_{11 \times 3}$. The factor importance weight set $\omega=\left(\omega_{1}, \omega_{2}, \ldots, \omega_{11}\right)$ was defined because the considered 11 variable factors were different effects on the final category evaluation, so the final category was synthetically evaluated according to fuzzy transformation for the evaluation matrix and the factor importance weight set.

The "high", "medium", "low" of the fuzzy sets were used to respectively describe the language discrete variables "security", "deashing ability", "dust-cleaning efficiency" and"stability and bearing of the structure", so the membership degree value can be calculated with the fuzzy statistics and expert evaluation method, as shown in Table 1 .

The language variables "filtration velocity","equipment resistance", "length of the filter bag", "service life of filter bag", "dust emission concentration" and "air volume","consumption of steel in the structure", are the continuous variables. The fuzzy concept reflected by the fuzzy sets of these continuous language variables can be described by the corresponding mature existed indicators. For example, the processing air volume between $3000 \mathrm{~m}^{3} / \mathrm{h}$ and $220000 \mathrm{~m}^{3} / \mathrm{h}$ was considered as the low processing air volume, the processing air volume between $220000 \mathrm{~m}^{3} / \mathrm{h}$ and $950000 \mathrm{~m}^{3} / \mathrm{h}$ was considered as the medium processing air volume, the processing air volume between $950000 \mathrm{~m}^{3} / \mathrm{h}$ and $2000000 \mathrm{~m}^{3} / \mathrm{h}$ was considered as the high processing air volume, so the continuous variable indicators were transformed into the membership function, and the membership function is adopted the straight ladder function and expressed expressed as Equation.2.

$$
u(x)=\left\{\begin{array}{cc}
0 & x \leq a_{1} \\
\frac{x-a_{1}}{a_{2}-a_{1}} & a_{1}<x \leq a_{2} \\
\frac{x-a_{2}}{a_{3}-a_{2}} & a_{2}<x \leq a_{3} \\
\frac{x-a_{3}}{a_{4}-a_{3}} & a_{3}<x \leq a_{4} \\
1 & x>a_{4}
\end{array}\right.
$$

Where $a_{i}$ and $a_{i+1}$ respectively express the segmentation boundary value in a certain section. And the segmentation boundary values of the membership function in various continuous language variables were shown in Table 2. According to user qualitative requirements for each continuous language variable, firstly, the membership functions of each factor fuzzy set were calculated, and the membership function of fuzzy concept was synthetically gotten, and then the fuzzy semantics of user requirements can be quantificationally analyzed through the relevant calculation of fuzzy set.

So, the fuzzy semantic model was established as follows:

Step1: The language variables of user requirements $\boldsymbol{U}=\left\{u_{1}, u_{2}, \ldots, u_{n}\right\}$ were determined to distinguish the discrete language variables and continuous language variables;

Step2: Supposing that the evaluation set was expressed as $\boldsymbol{V}=\left\{v_{1}, v_{2}, \ldots, v_{m}\right\}$, and gerenally $v_{l}$ expressed excellent, $v_{2}$ expressed good, $v_{3}$ expressed average, $v_{4}$ expressed poor;

Step3: The membership degree $r_{i j}$ was determined, which the single factor $u_{i}(i=1,2, \ldots, 11)$ in the domain $\mathrm{U}$ act on the category $v_{j}(j=1,2,3)$, and the total evaluation matrix $\mathbf{R}=\left(r_{i j}\right)_{n \times m}$ was constructed;

Step4: The effect weight of various factors in the final category evaluation was expressed as $\omega_{i}$, and then factor importance weight set $\omega=\left(\omega_{1}, \omega_{2}, \ldots, \omega_{11}\right)$ was determined;

Step5: The evaluation matrix and the factor importance weight set operated fuzzy transformation,

Table 1 The membership degree value of discrete language variable

\begin{tabular}{|c|c|c|c|c|c|c|c|c|c|c|c|c|c|}
\hline \multirow{2}{*}{$\begin{array}{c}\text { Requirements } \\
\text { category }\end{array}$} & \multicolumn{4}{|c|}{ Security } & \multicolumn{4}{c|}{ Deashing ability } & \multicolumn{3}{c|}{$\begin{array}{c}\text { Dust-cleaning } \\
\text { efficiency }\end{array}$} & \multicolumn{3}{c|}{$\begin{array}{c}\text { Stability and bearing } \\
\text { of the structure }\end{array}$} \\
\cline { 2 - 16 } & high & medium & low & high & medium & low & high & medium & low & high & medium & low \\
\hline $\begin{array}{c}\text { Classical } \\
\text { category }\end{array}$ & 0.2 & 0.2 & 0.7 & 0.1 & 0.2 & 0.5 & 0.1 & 0.2 & 0.5 & 0.1 & 0.15 & 0.8 \\
\hline $\begin{array}{c}\text { Improved } \\
\text { category }\end{array}$ & 0.3 & 0.35 & 0.2 & 0.35 & 0.4 & 0.3 & 0.35 & 0.4 & 0.3 & 0.2 & 0.25 & 0.15 \\
\hline $\begin{array}{c}\text { Innovation } \\
\text { category }\end{array}$ & 0.5 & 0.45 & 0.1 & 0.55 & 0.4 & 0.2 & 0.55 & 0.4 & 0.2 & 0.7 & 0.6 & 0.05 \\
\hline
\end{tabular}


the final category was synthetically evaluated according to the principle of the maximum membership degree.

Table 2 The segmentation boundary value of the membership function in various continuous language variables

\begin{tabular}{|c|c|c|c|c|}
\hline $\begin{array}{c}\text { Language } \\
\text { variable } x\end{array}$ & $\boldsymbol{a}_{\boldsymbol{1}}$ & $\boldsymbol{a}_{\mathbf{2}}$ & $\boldsymbol{a}_{\mathbf{3}}$ & $\boldsymbol{a}_{\boldsymbol{4}}$ \\
\hline $\begin{array}{c}\text { Filtration } \\
\text { velocity }(\mathrm{m} / \mathrm{min})\end{array}$ & 0.8 & 1.2 & 1.6 & 2.0 \\
\hline $\begin{array}{c}\text { Equipment } \\
\text { resistance }(\mathrm{Pa})\end{array}$ & 238 & 766 & 957 & 1342 \\
\hline $\begin{array}{c}\text { Length of the } \\
\text { filter bag }(\mathrm{m})\end{array}$ & 6 & 8 & 10 & 12 \\
\hline $\begin{array}{c}\text { Service life of } \\
\text { filter bag (year) }\end{array}$ & 2 & 3 & 4 & 5 \\
\hline $\begin{array}{c}\text { Dust emission } \\
\text { concentration } \\
\left.(\mathrm{mg} / \mathrm{Nm})^{3}\right)\end{array}$ & 10 & 8 & 6 & 2 \\
\hline $\begin{array}{c}\text { Air volume } \\
\left(\mathrm{m}^{3} / \mathrm{h}\right)\end{array}$ & 3000 & 220000 & 950000 & 2000000 \\
\hline $\begin{array}{c}\text { Consumption of } \\
\text { steel in the } \\
\text { structure }\left(\mathrm{kg} / \mathrm{m}^{2}\right)\end{array}$ & 30 & 35 & 40 & 45 \\
\hline
\end{tabular}

\subsection{Generate the concept set of bag filter product user requirements}

The outputs of fuzzy semantic model for user qualitative requirements are the user requirements category through the evaluation matrix and the factor importance weight set fuzzy transformation, and the weight and subset's property of the key associated factors under different user requirements categories. So, the classical category, improved category, and innovation category were gotten to construct the user requirements concept sets with the different function properties, as shown in Fig. 2, through the analysis of the users fuzzy semantic requirements for bag filter products. User requirements concept sets contains product terms, influence factors, and the corresponding relations between user requirements properties, product engineering parameters and specification parameters, etc, which are defined in the national standards under different requirements categories.

\subsection{Generate innovation concept solution of bag} filters product

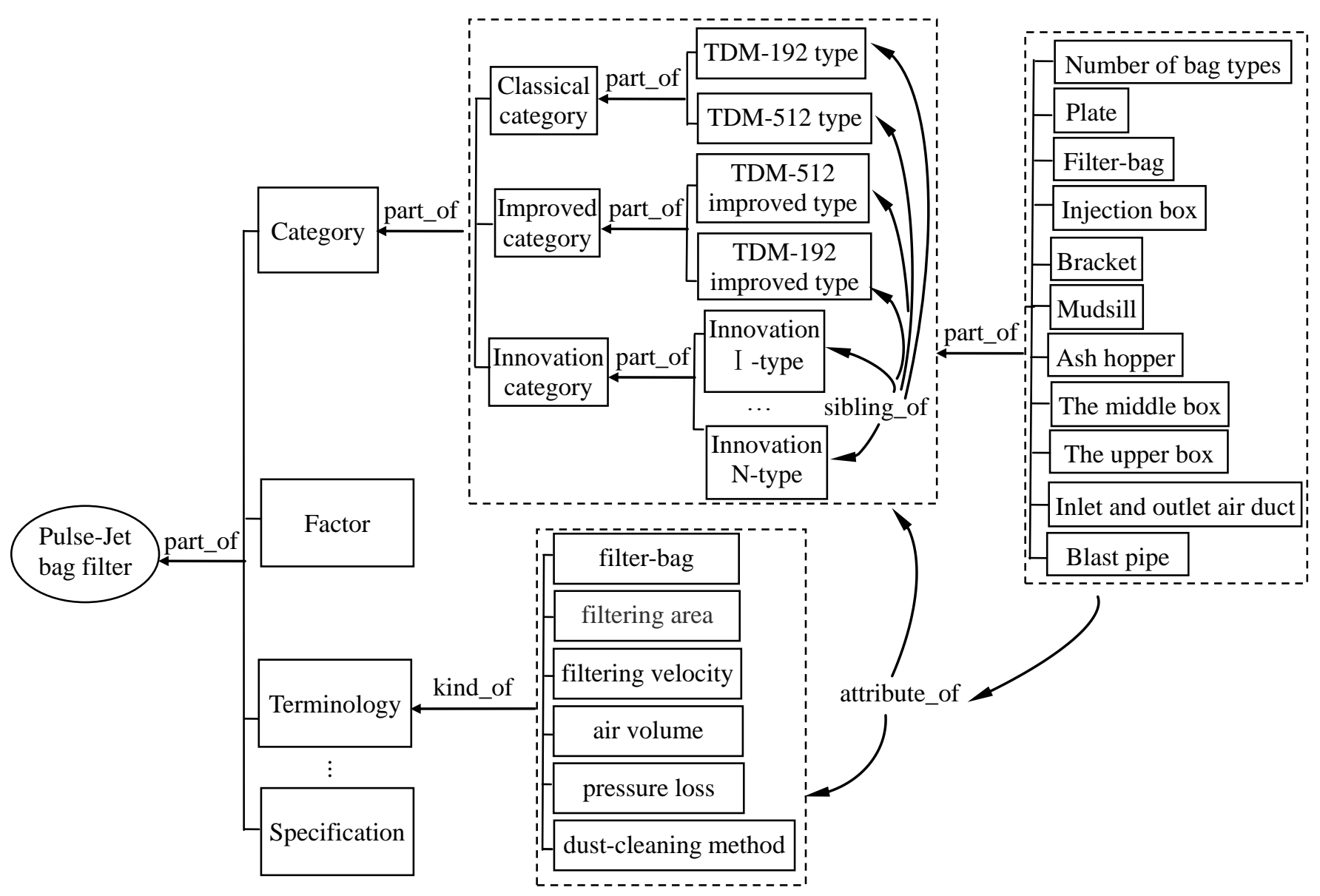

Figure 2 Concept set frame model of bag filter product user requirements. 
Three aspects of potential user qualitative requirements were predicted according to the rule of technology evolution, the fuzzy semantic model for the requirements was descripted, and the concept sets of user requirements were generated. The user requirements concept sets contain the product engineering characteristic parameters, influence factors, and user requirements properties corresponding relations under different requirements categories. The innovation solution was gotten in view of the user requirement easily dismantle and install on the site, which the plate valve was used instead of poppet valve in the upper box in order to save the space used to install the poppet valve, the low consumption of steel, and being easy to repair. The concept model was shown in Fig.3. The innovation solution was gotten in view of the user requirement that filter bag evolving to the direction of long bags and reducing the complexity of the filter bag structure, which the structure of "high injection net gas box" was used instead of the structure of "overhaul door \& injection box \& net gas chamber" in the upper box of the bag filter. In view of the fuzzy requirements that reducing the equipment resistance and injection pressure, improving the filtering speed, the deashing ability and dustcleaning efficiency, the corresponding correlation parameters were found, and the different inlet air ways and structures were found, for example under-inlet-type bag filter, side-down-inlet-type bag filter, face-into-inlet-type bag filter, divide the wind screen-type bag filter. And the different air inlet guiding devices were found, for example "no guiding device \& no inlet valve-type bag filter", "wind deflector \& no inlet valve-type bag filter", "trapezoidal plate \& no inlet valve-type bag filter", "diamond bluff body \& no inlet valve-type bag filter", "trapezoidal plate \& single plate valve-type bag filter", "no guiding device \& single plate valve-type bag filter","no guiding device \& multi plate valve-type bag filter". These structures have a greater influence on the filtering performance and air performance, the corresponding concept models of ash hopper, inlet pipe and the wind screen were gotten, as shown in Fig.4, 5, 6 respectively, the product innovation solutions were produced to guide the designer to realize the rapid innovation design, as shown in Fig.7.

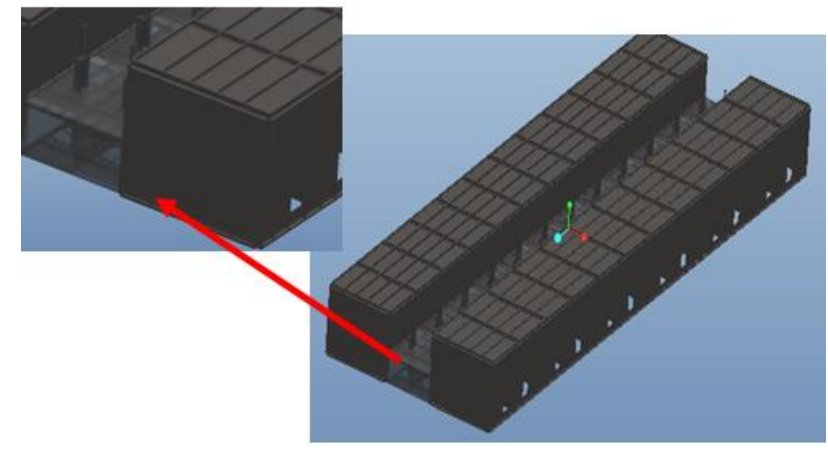

(a) Parallel angle type box \& poppet valve.

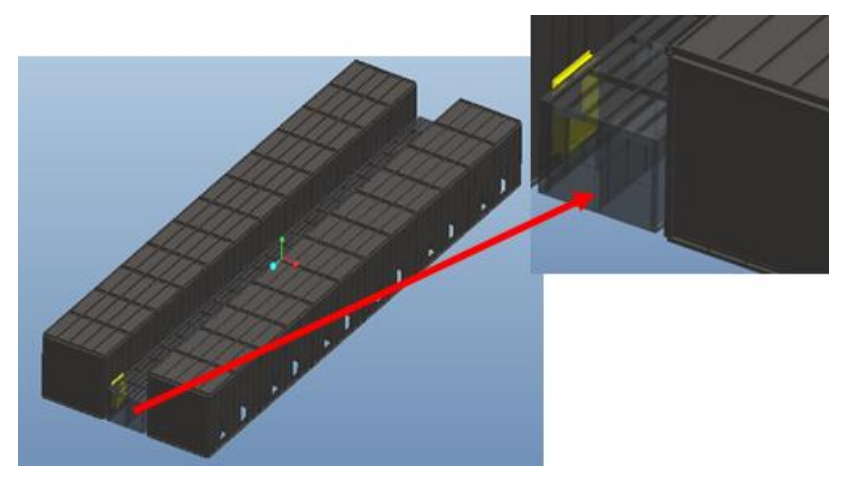

(b) Parallel angle type box \& plate valve.

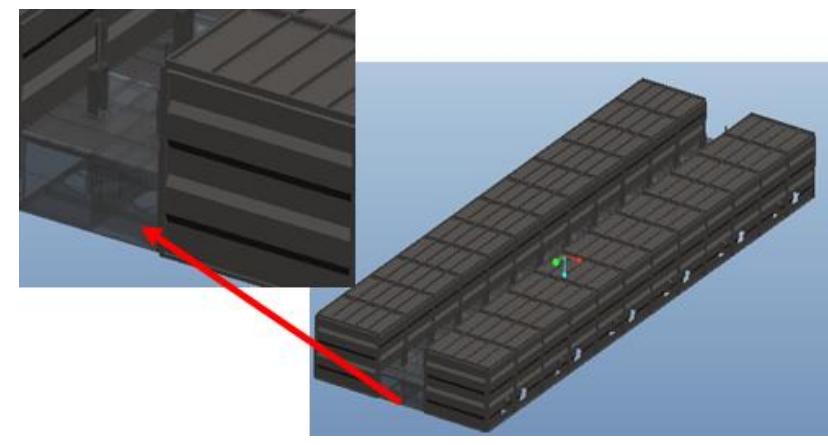

(c) Contour plate type box \& poppet valve.

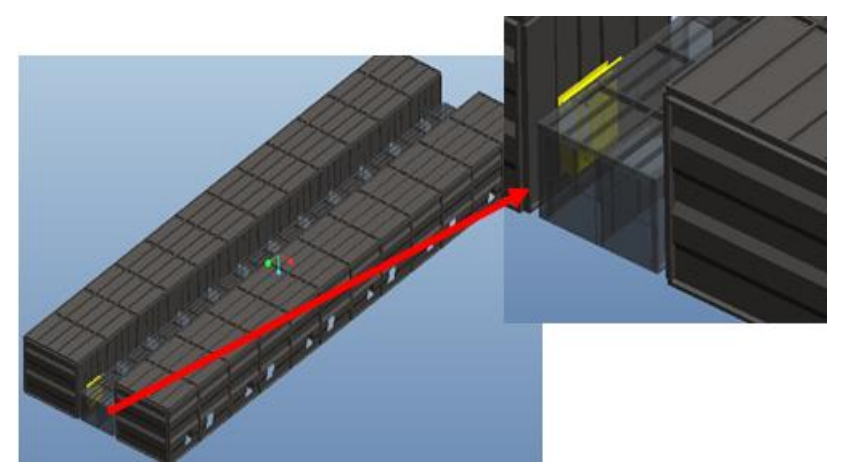

(d) Contour plate type box \& plate valve.

Figure 3 Concept model sets of the bag filter's upper box. 


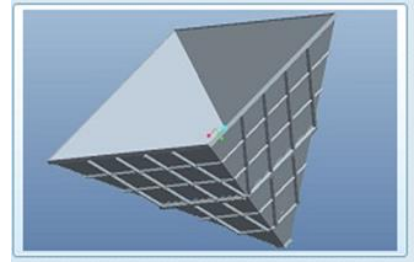

(a) No diversion-no inlet valve ash hopper.

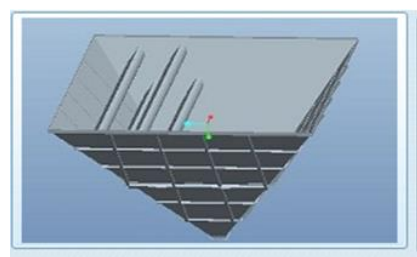

(c) Diamond blunt body-no inlet valve ash hopper.

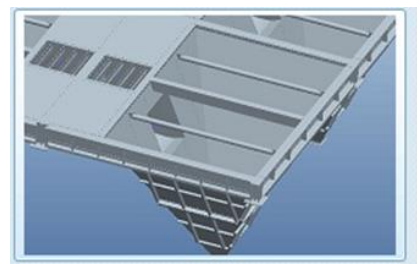

(e) Diversion- inlet valve 1 ash hopper.

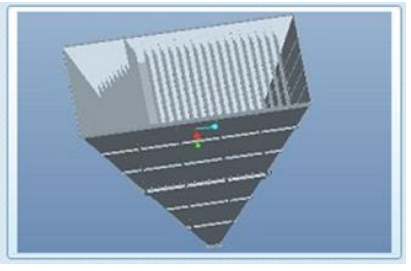

(b) Guide plate-no inlet valve ash hopper.

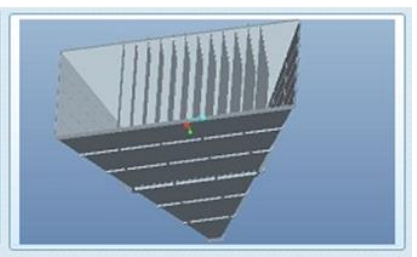

(d) Trapezoidal plate-no inlet valve ash hopper.

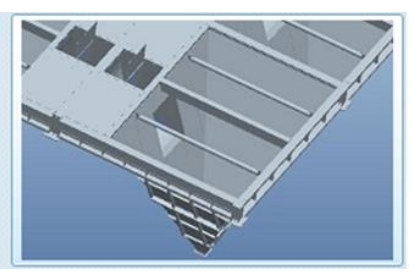

(f) Diversion- inlet valve2 ash hopper.
Figure 4 Concept model sets of the bag filter's ash hopper.

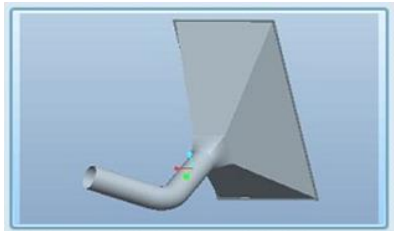

(a) Round elbow-lateral pipe.

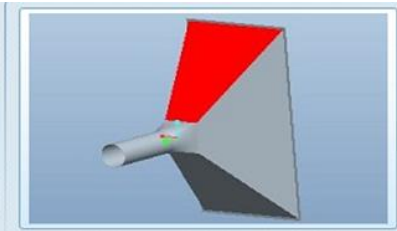

(b) Straight-forward pipe.

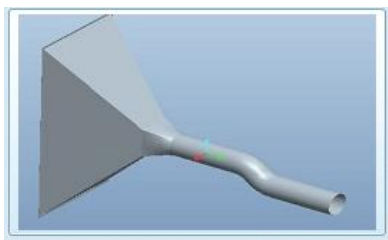

(c) S-linear-forward pipe.

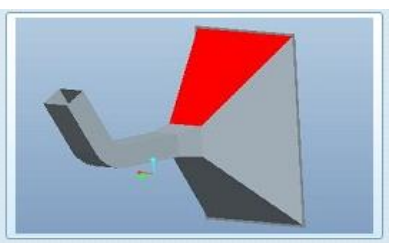

(d) Round elbow-lateral square pipe.

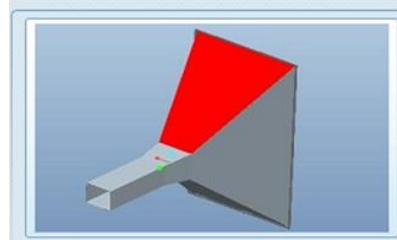

(e) Straight-forward square pipe.

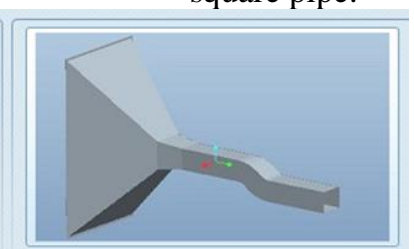

(f) S-linear-forward square pipe.
Figure 5 Concept model sets of the bag filter's blast pipe.

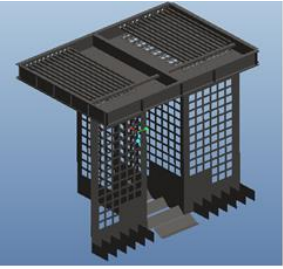

(a) T-type.

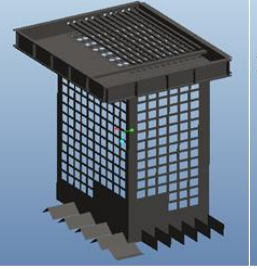

(b) L-type.

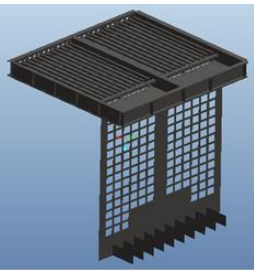

(c) I-type
Figure 6 Concept model sets of the bag filter's injection

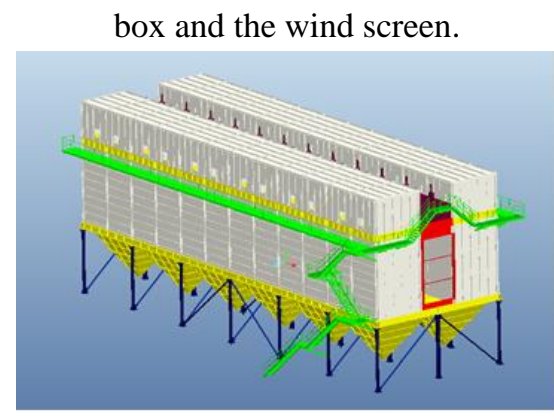

(a) Classical category TDM-192 type.

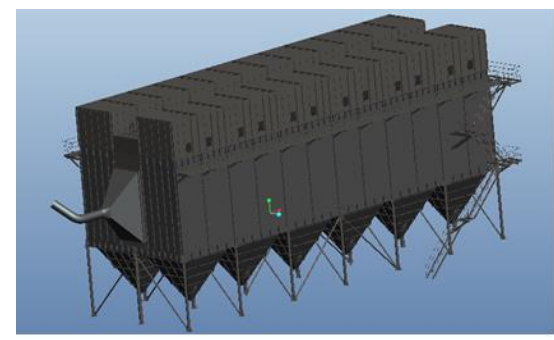

(b) Improved category TDM-192 type.

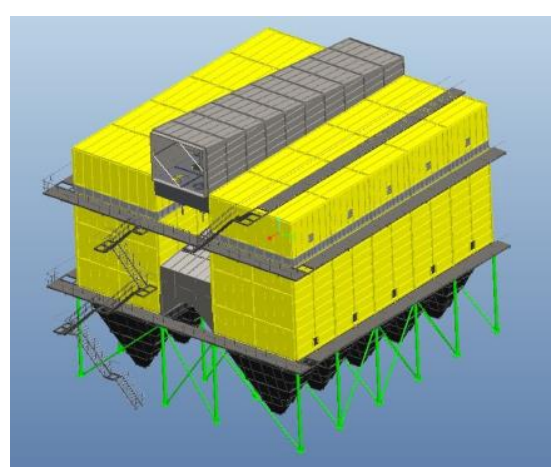

(c) Classical category TDM-512 type.

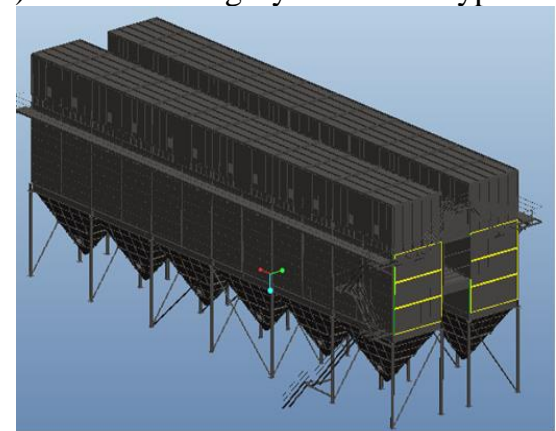

(d) Innovation category TDM-T type. 


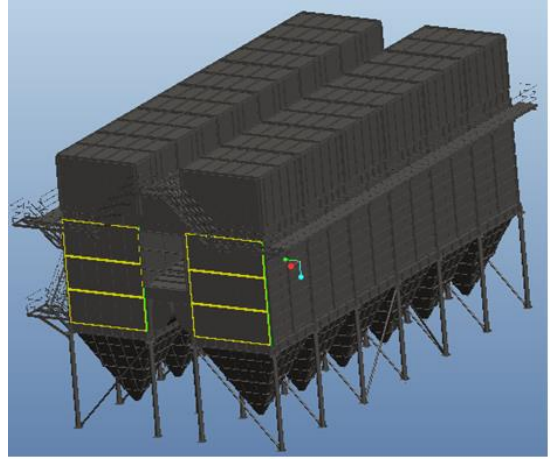

(e) Innovation category TDM-L type.

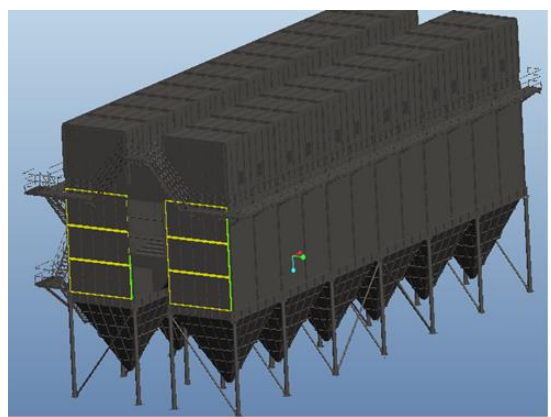

(f) Innovation category TDM-I type.

Figure 7 Innovation concept model of the bag filter.

\section{Conclusions}

The common classification methods of user requirements were analyzed, the changes of user functional requirements were analyzed combining with the product market requirements, and the trend of product functional requirements was judged, the user's potential requirements were predicted and elicitated with the technology evolution theory. In view of the fuzzy and uncertainty of users' potential requirements description, the user qualitative potential requirements were proposed based on the fuzzy semantic model, and the user requirements concept sets with functional attributes were standardly generated in different requirement categories. Finally, the effectiveness of the proposed method was verified taking the Pulse-Jet bag filter as an example, the method provide the unified model architecture for the mapping between subsequent innovation concept solutions and innovation function solutions and innovation structure solutions in the development of the new product. And it has certain significance to the variety user requirement analysis and generating of new products innovation ideas.

\section{Acknowledgments}

This project is supported by Tianjin Cement Industry Design \& Research Institute Co., Ltd. Post doctoral Project and National Natural Science Foundation of China (Grant No. 51305124) and Natural Science Foundation of Hebei Province (No. E2014202068)

\section{References}

[1] LIU Bin, ZHU Ming, WANG Jinghua, et al., "Research on customer requirement capturing ba sed on extension data mining", Journal of Hefei University of Technology (Natural Science), Vol.3 4, No.12, pp.1823-1826, 2011.

[2] TAN Runhua, "Progress of some problems in product design for innovation", Chinese Journal of Mechanical Engineering, Vol.39, No.9, pp.11-1 6, 2003.

[3] DING J, YANG D, BAO Z, "Research on Capturing of Customer Requirements Based on Innovation Theory", Physics Procedia, No.24, pp.18681880, 2012.

[4] WANG Y, CHIN K, “A linear goal programming approach to determining the relative importance weights of customer requirements in quality function deployment", Information Sciences, Vol.181, No.24, pp. 5523-5533, 2011.

[5] WANG Xiaotun, XIONG Wei, "Rough AHP approach for determining the importance ratings of customer requirements in QFD", Computer Integrated Manufacturing Systems, Vol.16, No.4, pp. 763-771, 2010.

[6] LI Yanlai, TANG Jiafu, PU Yun, et al., "Final importance ratings determining of customer requirements in quality function deployment", Com puter Integrated Manufacturing Systems, Vol.13, No.4, pp. 791-796, 2007.

[7] LI Zhaoling, GAO Qisheng, "Importance of customer requirements in quality function deployment based on two-tuple linguistic", Computer Integrated Manufacturing Systems, Vol.15, No.6, pp.1202-1206, 2009.

[8] WANG Yue, TSENG M, "Integrating comprehensive customer requirements into product design", CIRP Annals-Manufacturing Technology, Vol.60, No.1, pp. 175-178, 2011.

[9] ZHANG Heming, XIONG Guangleng, "Product requirements capturing and structurally modeling", Computer Integrated Manufacturing Systems, 
Vol.7, No.10, pp. 18-21, 2001.

[10]CHAN S, IP W,“A dynamic decision support system to predict the value of customer for new product development",Decision Support Systems, Vol.52, No.1, pp. 178-188, 2011.

[11]TAN Jiuanrong, FENG Yixiong, Design knowledge modeling, evolution and application, National Defense Industry Press, Beijing, pp.29-38, 2007.

[12]ZHANG Jianhui, TAN Runhua, ZHANG Peng, et al., "Process model of new ideas generation for product conceptual design by CAI", Computer Integrated Manufacturing Systems, Vol.19, No.2, pp. 284-292, 2013.

[13]Tan Runhua, Innovation design- TRIZ: Theory of Inventive Problem Solving, China Machine Press, Beijing, pp.53-75, 2006.

[14]LI Qingyong, HU Hong, SHI Zhiping, et al., "Re-search on texture-based semantic image retrieval", Chinese Journal of Computers, Vol.29, No.1, pp.116-123, 2006.

[15]NIE Daan, LI Yan, MA Guanglin, et al., "Simultaneous multi-product design method based on customer requirements classification", Computer Integrated Manufacturing Systems, Vol.16, No.6, pp.1131-1137, 2010.

[16]JIANG Weiguang,WU Jianwei,Wu Can, et al., "Ontology based product knowledge integration", Journal of Zhejiang University (Engineering Scien ce),Vol.43, No.10, pp.1801-1807, 2009.

[17]ZHANG Dianyin, WANG Chun, Pulse bag filter handbook, Chemical Industry Press, Beijing, pp.80-89,2011. 